\title{
The calculation of surface temperature and albedo of Arctic sea ice from AVHRR
}

\author{
R. LINDSAY AND D. ROTHROCK \\ Polar Science Center, Applied Physics Laboratory, University of Washington, Seattle, WA 98195, U.S.A.
}

\begin{abstract}
The temperature and albedo distributions of Arctic sea ice are calculated from images obtained from the AVHRR satellite sensor. The temperature estimate uses a split window correction incorporating regression coefficients appropriate for the arctic atmosphere. The albedo estimate is found assuming a clear and dry atmosphere. Both estimates are made with published correction techniques. Inherent errors due to the uncertainty of the atmospheric interference produced by humidity, aerosols, and diamond dust are judged to be $2-5^{\circ} \mathrm{C}$ in surface temperature and $0.10-0.20$ in surface albedo. Cloudy regions are masked out manually using data from all five channels. The relationship between temperature and albedo is shown for a sample scene. A simple model of a surface composed of only cold, bright ice and warm, dark water is inadequate. Model calculations based on the surface energy balance allow us to relate albedo and temperature to ice thickness and snow-cover thickness and to further assess the accuracy of the surface estimates.
\end{abstract}

\section{INTRODUCTION}

The Arctic is particularly sensitive to global climate change as indicated in the response of climate models to increased $\mathrm{CO}_{2}$ in the atmosphere (e.g. Manabe and Stoufer, 1980). Accurate estimates of both surface temperature and surface albedo are needed to provide input for climate models and to assess the degree of

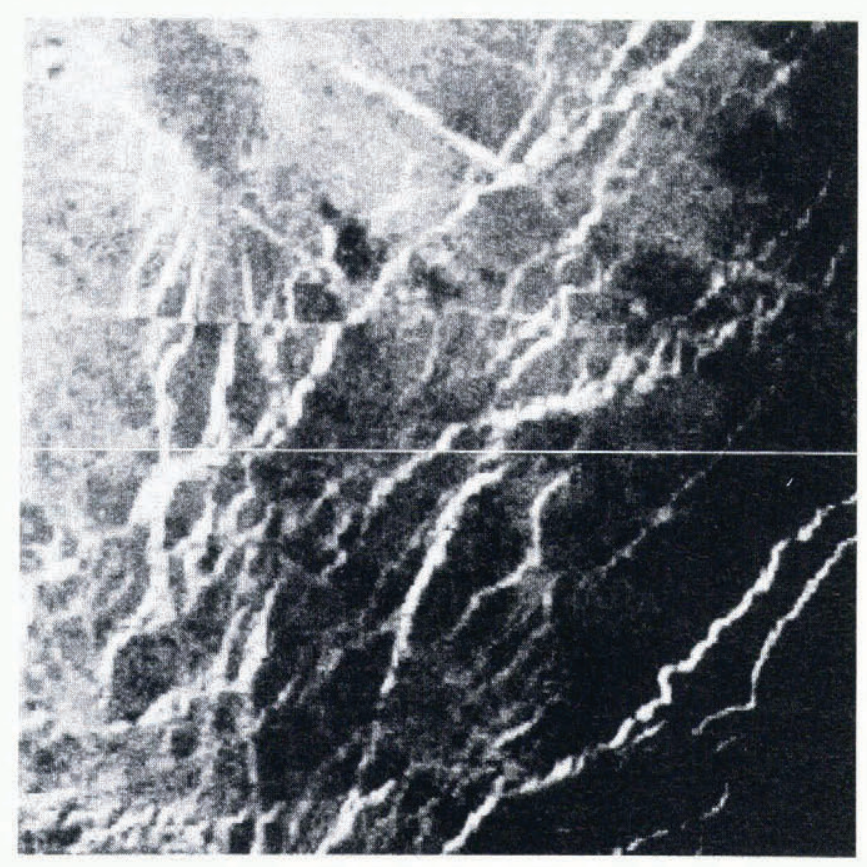

(a) climate change. Polar orbiting satellites have long provided data on these parameters in the polar regions, but only recently has sufficient computing power been available to provide estimates systematically over wide areas and for extended periods of time. We are assembling a database of cloud-masked images drawn from all regions of the Arctic Ocean and adjacent seas. Our first period of study is the year 1989, with studies including

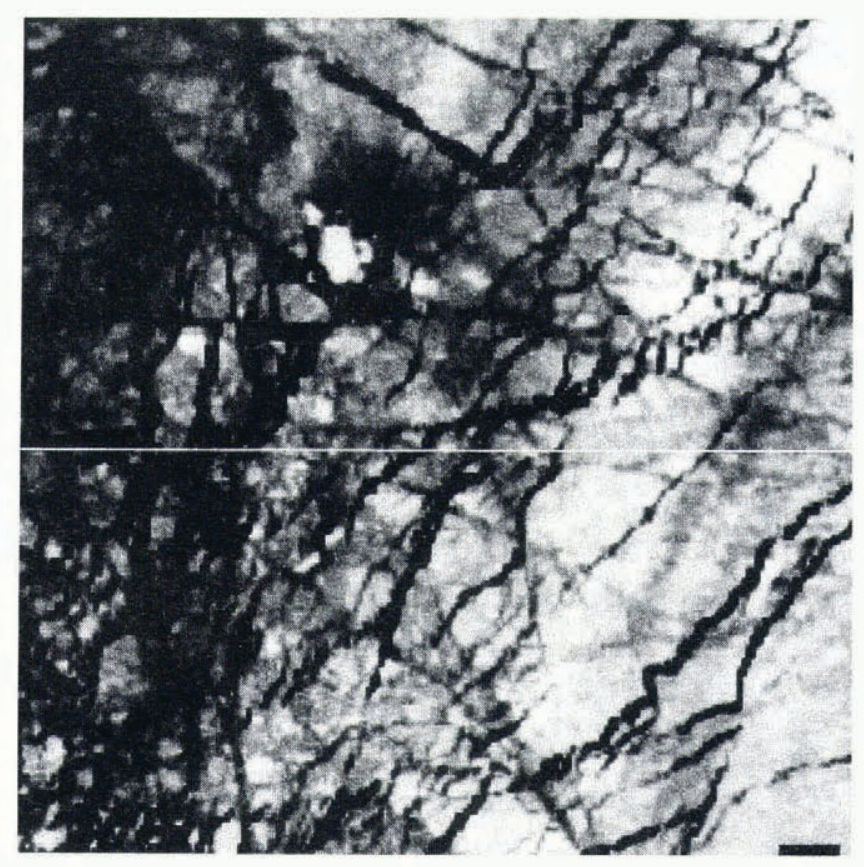

(b)

Fig. 1. $200 \mathrm{~km}$ AVHRR image. The images were acquired on 17 March 1989 at 2245 GMT in the Chuckchi Sea, centered at $69.6^{\circ} \mathrm{N}, 169.5^{\circ} \mathrm{W}$. The white line across the images corresponds to the transect plotted in Figure 2. (a) Surface temperature, cold is black, warm is white, range: $-20^{\circ}$ to $-5^{\circ} \mathrm{C}$. (b) Surface albedo, range: 0.1-0.85. 
additional years planned for the future. This paper describes our approach to acquiring temperature and albedo data from such imagery.

Temperature and albedo exhibit high spatial variability, with narrow leads appearing warm and dark, and multiyear ice floes appearing cold and bright. We describe some aspects of the distributions that we have found for the surface temperature and albedo over square regions, which we call cells, measuring $200 \times 200 \mathrm{~km}$. The size of these cells is a compromise among conflicting aims: including a significant number of leads in the scene, including a relatively homogeneous region of ice, and finding small cloud-free areas (often less than $200 \mathrm{~km}$ across).

In this paper we use an image from a region in the Chukchi Sea, dated 17 March 1989, to illustrate our procedure for estimating surface temperature and broadband albedo from the Advanced Very High Resolution Radiometer (AVHRR) radiances. The cell is characteristic of the central Arctic, consisting of multiyear and thick first-year ice with numerous leads. Figure 1 shows images of the estimated surface temperature and surface albedo, corrected for atmospheric interference.

The leads in AVHRR images are seldom wide enough to cover more than a few pixels. We rarely see leads that correspond to the "top hat" analogy, i.e. a constant temperature or albedo for thick ice broken by a relatively uniform warm, dark region. In Figure 2, transects of the temperature and albedo across the images in Figure 1 illustrate that, while leads certainly do appear as warm and dark areas, they do not appear as regions of constant temperature or albedo.

We review the corrections for atmospheric interference and discuss the univariate and bivariate distributions of the temperature and albedo. The bivariate distribution is compared with model estimates of the range of possible values. Future work will address the seasonal and regional variability of various temperature and albedo statistics (e.g. Lindsay and Rothrock, 1992).

The NOAA series of satellites are polar orbiting in sun-

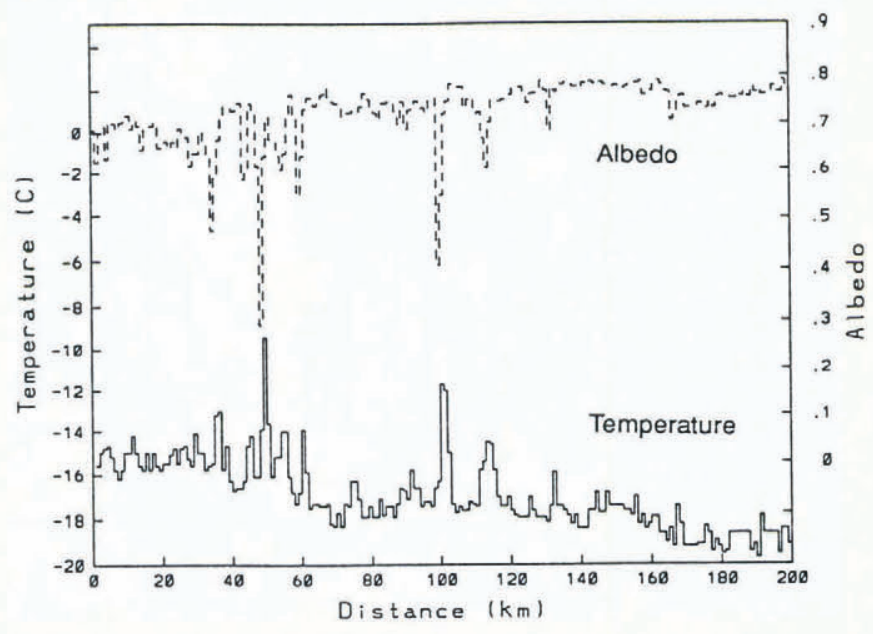

Fig. 2. Temperature and albedo transects along the horizontal line across the middle of the temperature and albedo images in Figure 1. synchronous orbits. The satellites carry the second AVHRR model, AVHRR/2. These radiometers come in two versions, one with five channels (on odd-numbered satellites plus NOAA-12) and the other with four (on even-numbered satellites). The five-channel version has two reflected solar channels ( 1 and 2), two thermal infrared channels (4 and 5) and one (3) that measures a combination of reflected solar radiation and emitted thermal radiation. The four-channel version is missing channel 5. The images we have processed, all for 1989, were obtained from NOAA-10 and 11. The orbital and instrumental characteristics are documented by Kidwell (1986).

\section{Geolocation and gridding procedures}

The process begins with level 1B data tapes obtained from the National Environmental Satellite Data and Information Service. Geolocation and calibration are performed at the Remote Sensing Branch, Naval Research Laboratory (NRL) at the Stennis Space Center in Mississippi. The calibration procedures include nonlinear corrections for channels 3, 4 and 5 but do not include corrections for the drift of the visible channels documented by Staylor (1990) and Abel (1990). The images are remapped to a polar stereographic grid, true at $70^{\circ} \mathrm{N}$, with a $1.0 \mathrm{~km}$ grid spacing. The orbital data are interpolated to the grid using a nearest-neighbor interpolation procedure, and the images are mapped to grids measuring $2250 \times 2800 \mathrm{~km}$. The complete procedures are described by Fetterer and Hawkins (1991) and Kidwell (1986).

Because of the variable resolution of the data across the image swath, the statistics calculated from the images are sensitive to the distance from the nadir track. Small leads are harder to detect, and high lead temperatures are less frequently encountered near the swath edges. The quantitative effect of the change in resolution depends on the spatial distribution of the underlying variable (temperature or albedo), and so it is generally impossible to predict. Because of the much lower resolution near the swath edge, we have limited our analysis to satellite zenith angles less than $45^{\circ}$ where the along-track resolution is $1.6 \mathrm{~km}$ and the cross-track resolution is $2.3 \mathrm{~km}$.

\section{Cloud masking}

There is not yet a reliable method for determining cloudy areas over pack ice from a single set of AVHRR images, although there has been a great deal of research on the problem (e.g. Ebert, 1989; Rossow, 1989; Welch and others, 1992). We are currently using a subjective cloud masking procedure that relies on both the thermal and visible channels. Subregions of the full images, measuring $512 \times 512 \mathrm{~km}$, are masked with an interactive program. In sunlit scenes, the channel 3 reflectance (approximated by channel 4 -channel 3 ) is useful along with the visible channel; at night, channel 4 and the difference between channels 4 and 5 or between 4 and 3 are often the only clues helpful in discerning cloud patterns. In all of the scenes there is some uncertainty in determining cloudy areas, which adds to uncertainties in the estimates of the surface temperature and albedo. Plumes on the lee side of leads are occasionally observed to extend many kilometers 
downwind of the leads: we may miss some plumes that are much smaller or less intense.

After the cloud masks are established, we select $200 \times 200 \mathrm{~km}$ square cells that are mostly cloud free and use them for our subsequent analysis. To date, we have cloud-masked 56 subregions and have selected 126 cells for analysis. Their distribution in time is irregular, falling mostly in January, March, July and October, but covers each of the seasons. Our present sample must be considered preliminary.

\section{Computation of surface temperature}

The ice surface temperature is estimated from channels 4 $(10.5-11.5 \mu \mathrm{m})$ and $5(11.5-12.5 \mu \mathrm{m})$ using a "split window" algorithm developed by Key and Haefliger (1992) that is specific for sea ice in the Arctic. Their algorithm is based on a radiometric model of the atmosphere applied to temperature and humidity data from radiosonde profiles obtained from Russian ice camps. The estimated surface temperature is given by

$$
T_{\mathrm{sfc}}=a_{0}+a_{4} T_{4}+a_{5} T_{5}+a_{45}\left(T_{4}-T_{5}\right) \sec \zeta
$$

where $T_{4}$ and $T_{5}$ are the brightness temperatures for channels 4 and $5, \zeta$ is the scan angle (angle from nadir at the satellite), and $a_{0}, a_{4}, a_{5}$ and $a_{45}$ are constants determined for each satellite and for each of three seasons. In our data the correction $T_{\text {sfc }}-T_{4}$ is positive and ranges from about 1 to about $4^{\circ} \mathrm{C}$; for moist tropical atmospheres the correction can be $10^{\circ} \mathrm{C}$ or more. Channel 5 is absorbed by the atmosphere slightly more than channel 4 . In our data, $T_{4}-T_{5}$ is typically $1^{\circ} \mathrm{C}$; the standard deviation within a cloud-free $200 \mathrm{~km}$ cell varies from $0.1^{\circ}$ to $0.4^{\circ} \mathrm{C}$.

The Key and Haefliger algorithm assumes a snow surface and includes the effects of the angular dependence of the emissivity of snow. The difference in emissivity between snow and water implies that this algorithm underestimates the temperature of water surfaces by up to $2.5 \mathrm{~K}$. This effect makes the apparent temperature of the water colder than that of the ice in the summer, when both are near the freezing point. The assumption that the surface is uniformly snow-covered is also challenged by the observation of clear lead patterns in images of $T_{4}-T_{5}$. The difference over some leads exceeds that over cold ice floes by about $1{ }^{\circ} \mathrm{C}$. This change in the channel difference may be partly due to more humidity over the leads, or may reflect different emissivities of young sea ice and snow.

NOAA-10 does not have channel 5 , so the split window technique cannot be applied. We have made a rough estimate of the surface temperature using the Key and Haefliger algorithm, coefficients for NOAA-11, and a fixed value of $T_{4}-T_{5}$ of $1.0^{\circ} \mathrm{C}$, a typical value for our data. These approximations introduce an additional 2$4^{\circ} \mathrm{C}$ of uncertainty in the surface temperature estimates from NOAA-10.

The uncertainty of the surface temperature estimate depends on the uncertainty in the state of the atmosphere and in the emissive properties of the surface. The Key and Haefliger algorithm does a good job of accounting for the variable humidity and temperature profiles of the atmosphere, but cannot account for the variable content of aerosols and diamond dust (low, very thin ice clouds). They found that their regression equation relating the modeled surface temperature to the modeled channels 4 and 5 brightness temperatures seen at the satellite (Equation (1)) has a very low r.m.s. error - less than $0.1^{\circ} \mathrm{C}$. This cannot, however, be taken as the accuracy of the estimate. The atmospheric effects of aerosols and diamond dust are not accounted for and may induce substantial errors of several degrees Celsius (Key and others, 1993). The small, suspended ice crystal particles, called diamond dust, are a common and important component of the radiative transfer processes in the Arctic (Curry and others, 1990). We judge the accuracy to be at best $1^{\circ}$ or $2^{\circ} \mathrm{C}$ and at worst $5^{\circ} \mathrm{C}$, but without surfacebased measurements it is impossible to verify the accuracy.

\section{Computation of surface albedo}

Channel 1 is in the visible portion of the spectrum (0.58$0.68 \mu \mathrm{m})$ and channel 2 is in the near infrared, just outside the visible band $(0.725-1.10 \mu \mathrm{m})$. (For completeness, channel 3 is $3.55-3.93 \mu \mathrm{m}$.) We wish to obtain the albedo $\alpha$ of the surface, defined as the proportion of the direct solar radiative flux reflected from the surface. The value reported in the NRL data for each channel $C_{i}(i=$ 1 or 2), is the reflectance of a perfectly reflecting Lambertian (isotropic) surface relative to an overhead sun. It is reported with a resolution of 0.001 . To estimate the equivalent isotropic surface albedo we must (1) include the effect of the reduction of the solar flux due to the sun angle, (2) correct for atmospheric interference, (3) convert the narrowband (channel) reflectance to a broadband reflectance, and (4) convert the reflectance measured from the satellite to one appropriate for the entire hemisphere above the pixel. The resulting broadband albedo is still a function of the solar zenith angle because snow and ice surfaces reflect more radiation at low sun angles.

There is a significant drift in the instrument calibrations of the albedo channels that can be compensated for with time-dependent calibration coefficients appropriate to each satellite. These coefficients have been estimated for NOAA-6, 7, and 9 (Staylor, 1990), but have not yet been established for NOAA-10 and 11 and so are not included here. The drift can be quite significant. Staylor found degradation rates for the calibration coefficient of NOAA $-6,7$, and 9 on the order of $0 \%, 3.5 \%$ and $6.0 \%$ per year for channel 1. Without estimates of the instrument degradation rates, we have assumed no degradation; however, we acknowledge that the uncertainty is increased and we may be underestimating the true reflectance.

Accounting for the solar zenith angle gives us the planetary (top-of-atmosphere) reflectance for each channel:

$$
r_{i}(\theta, \phi, \xi)=\frac{C_{i}}{\cos (\theta)}, \quad i=1,2
$$

where $\theta$ is the solar zenith angle, $\phi$ is the satellite zenith angle and $\xi$ is the sun-satellite azimuth for each pixel.

Koepke (1989) developed a method for estimating the surface reflectance $R_{i}$ from the top-of-atmosphere 
reflectance $r_{i}$ specific to each AVHRR channel. It is a linear relationship with coefficients $a_{i}$ and $b_{i}$ that depend on the solar zenith angle, the optical depth of the atmosphere due to aerosols, the integrated ozone content of the atmosphere for channel 1, and the water vapor content for channel 2:

$$
R_{i}(\theta, \phi, \xi)=\frac{r_{i}(\theta, \phi, \xi)-a_{i}(\theta)}{b_{i}(\theta)} .
$$

In our analysis we have assumed the skies are quite clear (optical depth for aerosols $=0.05$ ) and dry (water vapor content $=0.5 \mathrm{~g} \mathrm{~cm}^{-2}$ ) with a low concentration of ozone $(0.24 \mathrm{~cm} \mathrm{NTP})$, for lack of any specific data for these parameters. These assumptions minimize the atmospheric correction; the effect of the corrections is to add 0.04 and 0.08 to the mean reflectance of channels 1 and 2 for our sample scene. If the atmosphere were very hazy, as is often observed in the Arctic, very dark surfaces would appear brighter due to back-scattered radiation from the aerosols, and very bright surfaces darker due to absorption of radiation by the aerosols. Contrast between leads and floes would be reduced (Key and others, 1993). If the coefficients that Koepke suggested for a hazy atmosphere (optical depth for aerosols $=0.4$ ) are used for the scene in Figure 1a, but we still assume a dry atmosphere with low ozone levels, the estimated albedo of the bright areas is increased by 0.15 , while that of the dark areas is reduced by 0.10 . The unknown haze content (optical depth) of the atmosphere and the unknown moisture and ozone content introduce an uncertainty in the albedo estimates that may amount to 0.20 . The atmospheric corrections, and the uncertainties, are larger for lower sun angles and also for very dark surfaces (water) and very bright surfaces (snow) than for moderately bright surfaces (bare ice or frozen leads).

The broadband albedo can be estimated using the two narrowband measurements. Wydick and others (1987) suggested regression equations for different surface types, as well as global equations. They use two different methods, one based on a radiative transfer model of the atmosphere, and one based on comparing narrowband AVHRR measurements with Earth Radiation Budget (ERB) measurements. We have used their equation for snow, derived from the radiative transfer model, to estimate the broadband reflectance:

$$
R(\theta, \phi, \xi)=0.0047+0.40 R_{1}(\theta, \phi, \xi)+0.43 R_{2}(\theta, \phi, \xi)
$$

Strictly speaking, this correction relates to a top-of-theatmosphere reflectance, but we have assumed it is also appropriate for the clear-sky surface reflectance.

Radiation impinging on a surface at a solar zenith angle $\theta$ is reflected into all angles $(\phi, \xi)$ in the hemisphere above the surface, as specified by the bidirectional reflectance function $\operatorname{BDRF}(\theta, \phi, \xi)$. The surface albedo is the integral of this reflectance over the hemisphere

$$
\alpha(\theta)=\frac{1}{\pi} \int_{0}^{\pi / 2} \int_{0}^{2 \pi} \operatorname{BDRF}(\theta, \phi, \xi) \cos \phi \sin \xi \mathrm{d} \xi \mathrm{d} \phi .
$$

(We denote by $\mathrm{R}$ the observed reflectance at a particular viewing geometry, and by BDRF the reflectance at all viewing angles; these are the same quantity.) We want to use a single observation of reflectance ( $R$ in Equation (4)) from the satellite at a known viewing geometry $(\phi, \xi)$ and known solar zenith angle $\theta$ to estimate the albedo. To do so we need to know the relationship between the reflectance at a particular viewing angle and the total surface reflectance $\alpha$. The ratio of $R$ or BDRF to albedo is the anisotropic reflectance factor $f$; it can be thought of as the normalized BDRF; it integrates over the hemisphere to unity.

$$
f(\theta, \phi, \xi)=\frac{\operatorname{BDRF}(\theta, \phi, \xi)}{\alpha(\theta)}
$$

The value of $f$ has been compiled by Taylor and Stowe (1984) for a variety of different surfaces using ERB data. We use a smoothed version of their estimates of $f$ for sea ice to convert the estimate of the observed broadband reflectance, $R$, to obtain broadband albedo:

$$
\alpha(\theta)=\frac{R(\theta, \phi, \xi)}{f(\theta, \phi, \xi)}
$$

The smoothed $f$ ranges from 0.6 to 1.1 . The albedo is still a function of the solar zenith angle because of the increase in the albedo of snow for low sun angles (Taylor and Stowe, 1984). The albedo was determined for any cell with a solar zenith angle less than $80^{\circ}$ (i.e. sun more than $10^{\circ}$ above the horizon). The use of a snow surface for the broadband albedo and the bidirectional reflectance corrections may introduce errors in the albedo estimates for thin ice and open water areas.

The overall accuracy of the broadband albedo estimate is affected by the corrections we made: (1) for atmospheric interference, (2) for narrowband to broadband conversion, and (3) for the bidirectional reflectance. Errors may also arise from (4) unknown drift in the instrumental calibration and (5) uncertainty in cloud masking. With all of these sources of error, it is not

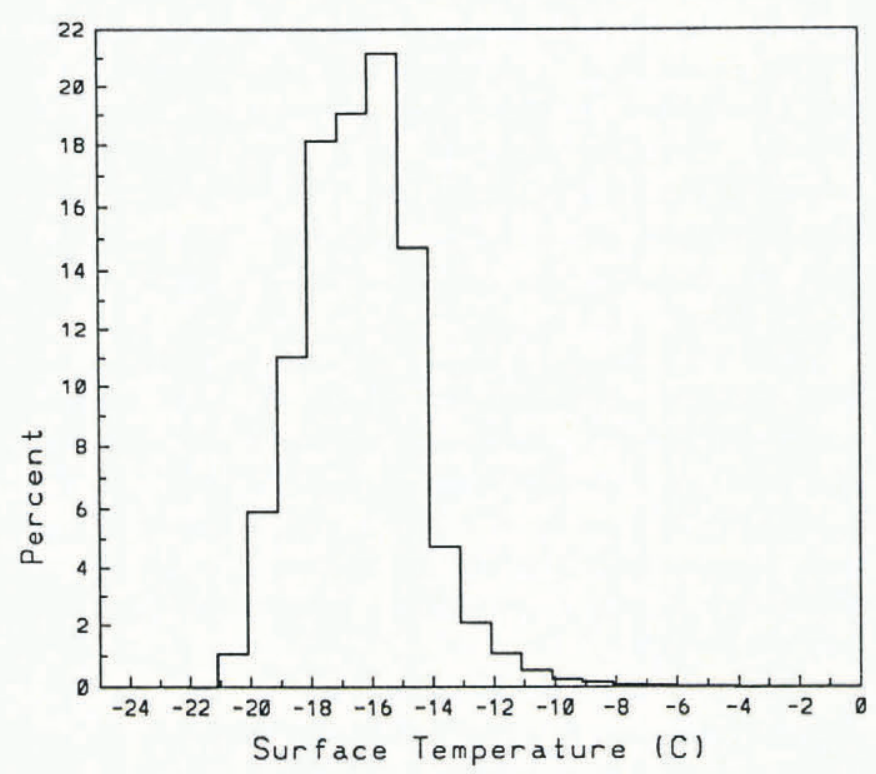

Fig. 3. Histograms of the temperature distribution from the image in Figure 1a. 
possible to place definitive error bars on our surface albedo estimate; we feel that in most scenes the estimate is within 0.1 , but in some cases the uncertainty can amount to 0.2 .

\section{SURFACE TEMPERATURE AND ALBEDO DIS- TRIBUTIONS}

\section{Temperature}

The distribution of the temperature within a cell is usually strongly peaked with a warm tail representing leads and first-year ice. A histogram of the temperature distribution for the image in Figure $1 \mathrm{a}$ is shown in Figure 3. Because of the low resolution of the sensor compared with typical lead sizes, AVHRR images rarely show a bimodal distribution, where the two modes correspond to thick ice and leads. On the occasions when a bimodal distribution is observed, we commonly find that the image cell is composed of two regimes, one part mostly multiyear ice and the other part mostly thin first-year ice. The mean surface temperature estimate over a single cell in our sample ranges from $-46.3^{\circ} \mathrm{C}$ in March to $+0.4^{\circ} \mathrm{C}$ in June. The standard deviation within a cell ranges from $0.2^{\circ} \mathrm{C}$ in June to $4.7^{\circ} \mathrm{C}$ in January. The standard deviation is largest for the moderately cold scenes. The temperature variability over individual ice floes is quite small, ranging $1^{\circ}$ or $2^{\circ} \mathrm{C}$ over $10-20 \mathrm{~km}$.

There is often a significant variation in the mean temperatures of the large floes across the image. This variation may arise from several sources: different parts of the scene may have been cloud free for a longer period of time than other parts, may be differentially influenced by advective air flow, or may be differentially influenced by haze or diamond dust. In addition, part of the scene may include floes with somewhat thinner ice or more snow cover than other parts. We estimate a constant gradient across the scene by dividing the cell into $3 \times 3$ equally sized regions, finding the coldest quartile of the temperature for each region, and fitting a plane to these nine temperatures. Determined in this way, the "background" gradient is largely independent of the existence and the position of warm leads. This large-scale gradient is typically less than 2 deg per $100 \mathrm{~km}$ but ranges up to $6 \mathrm{deg}$ per $100 \mathrm{~km}$. The standard deviation of the temperature, with the background gradient removed, is reduced by 10 to $50 \%$. The background temperature gradient of about $2.4 \mathrm{deg}$ per $100 \mathrm{~km}$ can be discerned in the image in Figure 1a and in the transect in Figure 2.

\section{Albedo}

The albedo also shows a skewed distribution with a dark tail corresponding to leads. Bimodal distributions are almost never seen in the $200 \mathrm{~km}$ cells we have analyzed. A histogram of the albedo distribution for the image in Figure $1 \mathrm{~b}$ is shown in Figure 4. In our sample, the mean albedos range from 0.20 in a June cell of low ice concentration to 0.84 in a March cell. The maximum values, representative of floes with no contamination from leads, range from 0.27 for a June cell to 0.97 for a March cell. Standard deviations of the albedo within one scene

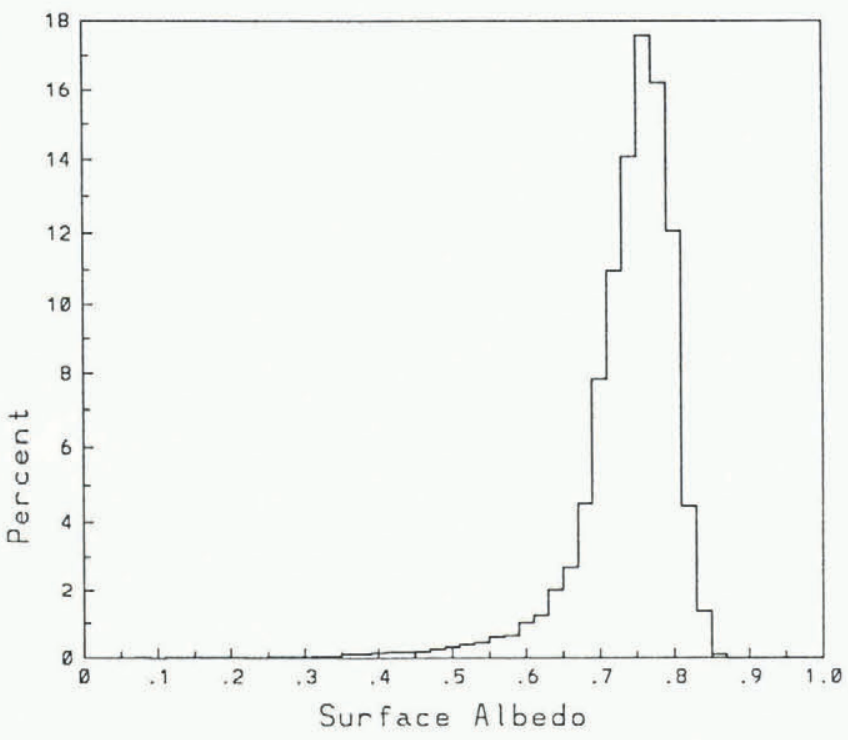

Fig. 4. Histograms of the temperature distribution from the image in Figure $1 b$.

ranged up to 0.15 , with a wide range of values seen in all seasons. For comparison, typical albedo values reported in the literature are $0.10-0.30$ for water, $0.15-0.40$ for melt ponds, $0.55-0.70$ for bare ice at freezing, $0.70-0.75$ for bare ice below freezing, and $0.80-0.85$ for cold, snowcovered thick ice.

The background gradients are determined in a manner similar to that for temperature. We fit a plane to the brightest quartiles of the nine albedo distributions. The gradients range up to 0.15 per $100 \mathrm{~km}$ but are typically less than 0.05 per $100 \mathrm{~km}$. The albedo gradient in Figure 2 is 0.05 per $100 \mathrm{~km}$. The cause of these gradients is a puzzle. There may be a residual correction for the solar zenith angle that has not been correctly applied or a non-uniform atmospheric haziness that is unaccounted for. At the high solar zenith angles that are common in the Arctic it is difficult to be sure of the cause.

\section{Bivariate distribution}

Clearly the albedo and temperature of the ice are strongly related. Open water is relatively warm and dark, while thick ice is cold and bright. Thin ice and leads are in between. We need a more complete understanding of the relationship for two reasons. First, in studies of leads, for example, we want to alternate between using the albedo in summer (when the surface is at the freezing point) and the temperature in the winter (when there is not enough sun to observe the albedo). Second, in spring and fall, when both temperature and albedo are available, we can use the two variables to deduce more information about the ice and snow.

The correlation of temperature and albedo is commonly negative, ranging to -0.89 . The correlation for the sample scene (with the background gradients removed) is -0.80 . In summer, when the lower emissivity of the water makes the open water look colder than the ice, the correlation can become positive. 


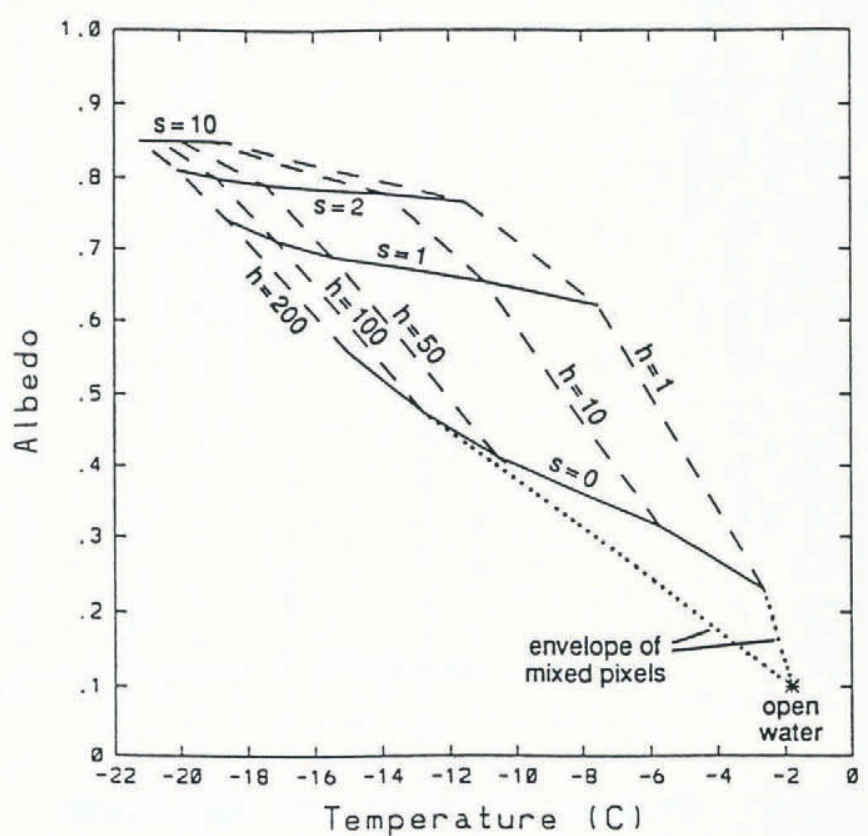

Fig. 5. Schematic of ice thickness and snow-depth effects on albedo and temperature plot. Model parameters include air temperature $=-15^{\circ} \mathrm{C}$, wind speed $=5 \mathrm{~m} \mathrm{~s}^{-1}$, downward longwave radiative flux $=100 \mathrm{Wm}^{-2}$, and downward shortwave flux $=370 \mathrm{Wm}^{-2}$. Dashed lines correspond to constant ice thickness $h$, in centimeters; solid lines correspond to constant snow depth $s$, in centimeters. The open water point $\left(T=-1.8^{\circ} \mathrm{C}, \alpha=0.1\right)$ is marked with an astarisk. The dotted lines indicate the envelope within which all mixed pixels should lie.

A simple, one-dimensional thermodynamic model of the ice with a snow layer allows us to predict the surface temperature and albedo for a given ice thickness $h$, snow depth $s$, air temperature, wind speed, and incoming longwave and shortwave radiative fluxes. The model follows the thin-ice thermodynamic model of Maykut (1978) and is based on a surface heat-balance equation and a steady-state thermal regime. Figure 5 is a plot of the model output of albedo vs temperature for various ice thicknesses (dashed line) and snow depths (solid line). Because the albedo of thin ice $(h=1 \mathrm{~cm})$ is substantially greater than that of open water, the line corresponding to bare ice $(s=0)$ does not tend to converge on the open water point. This diagram is appropriate for pure pixels, with no mixtures of ice of various thicknesses or snow of various depths. In a satellite image we would expect mostly mixed pixels for the warm and dark areas that indicate leads. Mixed pixels that include variable proportions of two distinct surfaces would appear on a straight line connecting any two points in the temperature-albedo plane. We would expect all of the pixels from a scene to lie within the convex hull that encompasses the open water point and all of the ice thickness and snow depth combinations, as shown by the dotted lines extending to the open-water point.

Figure 6 shows a scattergram of the pixel values of broadband surface albedo and of surface temperature for the sample scene shown in Figure 1 (17 March 1989,

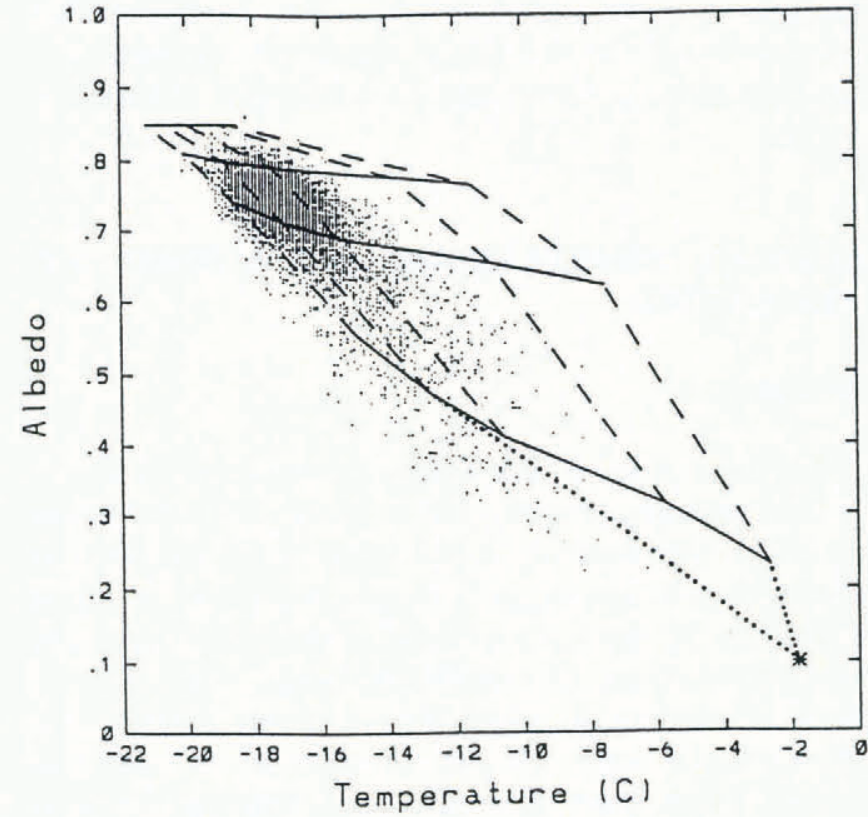

Fig. 6. Albedo vs temperature from the image in Figure 1, with the effect of the background gradient removed (no correction on the middle of the scene). The lines correspond to the model results shown in Figure 5.

Chukchi Sea). The air temperature for the model has been chosen to produce the observed approximate surface temperature for the bulk of the points, the cold and bright, thick ice. The model solar radiation is computed from the solar zenith angle, and the wind speed and downward longwave radiation are climatological values. We see that most of the points are concentrated in the cold and bright portion of the graph, corresponding to thick ice, with a few tending toward the open water point. There is a great deal of scatter, indicating that the ice cover is not a simple mixture of thick ice and open water but a complex mixture of thick ice, thin ice of various thicknesses and snow depths, and open water. We also note that the amount of snow shown for the bulk of the points, $1-2 \mathrm{~cm}$, is less than would be expected for the central Arctic.

The fact that all of the points do not fall within the envelope of expected values can be explained in a combination of modeling and measurement errors. First, the model may not be correct in its formulation or in its input parameters. The model is steady state and does not allow for a diurnal cycle or changeable cloud cover. This assumption will produce the largest errors in the thick ice and smaller errors in the thin ice which can reach thermal equilibrium faster. We have also used climatological values of the wind speed and the clear-sky, downward, shortwave radiative flux. Lower wind speeds would reduce the sensible heat flux from thin ice and allow for warmer thin-ice temperatures. Stronger, downward, longwave radiative flux would also allow for warmer thin ice, but would warm the thick ice as well. Secondly, the estimated surface temperature and albedo values may be incorrect. The most likely source of error is unaccounted-for haze or diamond dust (Curry and 
others, 1990). Haze would make the darker leads appear too cold and bright. Using the atmospheric correction coefficients (from Koepke, 1989) appropriate for a very hazy atmosphere (optical depth $=0.4$ ) in a test case, we find that the estimated surface albedo for the bulk of the points rises from $\alpha=0.80$ to $\alpha=0.95$, and the darkest points become darker, from $\alpha=0.25$ to $\alpha=0.15$. We have not yet examined the haze-related corrections to the surface temperature.

\section{CONGLUSIONS}

The excellent coverage of polar regions by NOAA satellites provides great motivation for routine estimation of clear-sky albedo and temperature from AVHRR. Applying standard corrections and conversions, we compute the surface temperature and broadband albedo. We estimate the uncertainty in temperature to be about $5^{\circ} \mathrm{C}$, and in albedo to be about $0.1-0.2$. These large errors limit the value of this data source for climate change studies. For example, a reduction in the albedo of bare summer ice from 0.64 to 0.54 would cause enough ablation to reduce the annual mean ice thickness by more than 50\% (Maykut and Untersteiner, 1971).

The sources of error include an inability to recognize thin clouds and haze, and, for albedo, instrument calibration uncertainties and use of climatological instead of instantaneous data about atmospheric humidity and ozone. Not all clouds can be detected and masked, especially at night when channel 3 reflectance is not available. In addition, there may be small clouds on the lee side of leads that go undetected yet bias the measurements. Aerosols and diamond dust are also undetectable and interfere with the satellite's view of the surface.

In spite of the obvious problems with AVHRR estimates of surface temperature and albedo, these data are useful. A clearly unique application is the use of spatial variability on $1 \mathrm{~km}$ scales to provide quantitative information about leads and polynyas. We have illustrated relating temperature and albedo to snow depth and the thickness of thin ice; with additional data on haze and atmospheric humidity to reduce the errors, these estimates would prove quite valuable.

\section{ACKNOWLEDGEMENTS}

This work is supported under Office of Naval Research grant number N00014-90-J-1074. We are grateful to F. Fetterer and the Remote Sensing Branch of the Naval Research Laboratory for preparing the AVHRR data and to J. Francis for the use of the computer code for the thermodynamic model of sea ice.

\section{REFERENCES}

Abel, P. 1990. Prelaunch calibration of the NOAA-11 AVHRR visible and near IR channels. Remote Sensing Environ., 31, 227-229.
Curry, J. A., F. G. Meyer, L. F. Radke, C. A. Brock and E.E. Ebert. 1990. Occurrence and characteristics of lower tropospheric ice crystals in the Arctic. Int. 7 . Climatol., 10(7), 749-764.

Ebert, E. E. 1989. Analysis of polar clouds from satellite imagery using pattern recognition and a statistical cloud analysis scheme. J. Appl. Meteorol., 28, 382-399.

Fetterer, F. M. and J. D. Hawkins. 1991. An AVHRR data set for the Arctic leads ARI. Naval Oceanographic and Atmospheric Research Laboratory. Technical Note 118.

Key, J. and M. Haefliger. 1992. Arctic ice surface temperature retrieval from AVHRR thermal channels. 7. Geophys. Res., 97(D5), 5885-5893.

Key, J., R. Stone, J. Maslanik and E. Ellefsen. 1993. The detectability of sea-ice leads in satellite data as a function of atmospheric conditions and measurement scale. Ann. Glaciol., 17 (see paper in this volume).

Kidwell, K. B., ed. 1986. NOAA polar orbiter data users guide. Washington, DC, U.S. Department of Commerce. National Oceanic and Atmospheric Administration. Satellite Data Services Division. (Revised 1988, 1991.)

Koepke, P. 1989. Removal of atmospheric effects from AVHRR albedos. F. Appl. Meteorol., 28, 1341-1348.

Lindsay, R. and D. Rothrock. 1992. The spatial structure of the surface temperature field of Arctic pack ice determined with AVHRR. Proceedings of the American Meteorological Society 3rd Conference on Polar Meteorology and Oceanography, Portland, OR, September 1992, 76-79.

Manabe, S. and R.J. Stouffer. 1980. Sensitivity of a global climate model to an increase of $\mathrm{CO}_{2}$ concentration in the atmosphere. F. Geophys. Res., 85(C10), 5529-5554.

Maykut, G. A. 1978. Energy exchange over young sea ice in the central Arctic. 7. Geophys. Res., 83(C7), 36463658.

Maykut, G. A. and N. Untersteiner. 1971. Some results from a time-dependent thermodynamic model of sea ice. 7. Geophys. Res., 76(6), 1550-1575.

Rossow, W.B. 1989. Measuring cloud properties from space: a review. 7. Climate, 2, 201-213.

Staylor, W.F. 1990. Degradation rates of the AVHRR visible channel for the NOAA 6,7 , and 9 spacecraft. 7 . Atmos. Oceanic Technol., 7, 411-423.

Taylor, V.R. and L. L. Stowe. 1984. Atlas of reflectance patterns for uniform Earth and cloud surfaces (NIMBUS-7 ERB-61 days). NOAA Tech. Rep. NESDIS 10.

Welch, R. M., S. K. Sengupta, A.K. Goroch, P. Rabindra, N. Rangaraj and M. S. Navar. 1992. Polar cloud and surface classification using AVHRR imagery: an intercomparison of methods. F. Appl. Meteorol., 31, 405-420.

Wydick, J.E., P.A. Davis and A. Gruber. 1987. Estimation of broadband planetary albedo from operational narrowband satellite measurements. NOAA Tech. Rep. NESDIS 27.

The accuracy of references in the text and in this list is the responsibility of the authors, to whom queries should be addressed. 